\title{
An Analysis for the Questionnaire Investigation about the High School Teachers Workload
}

\author{
Yasuo Ishii ${ }^{1}$, Tatsuya Oyanagi ${ }^{2}$, Daisuke Takeyasu ${ }^{3} \&$ Kazuhiro Takeyasu $^{4}$ \\ 1Yamato University, Japan \\ ${ }^{2}$ Hachinohe Gakuin University, Japan \\ ${ }^{3}$ The Open University of Japan, Japan \\ ${ }^{4}$ Tokoha University, Japan \\ Correspondence: Kazuhiro Takeyasu, College of Business Administration, Tokoha University, 325 Oobuchi, Fuji \\ City, Shizuoka 417-0801 Japan. Tel: 81-545-36-1133
}

Received: May 2, 2016

Accepted: July 5, 2016

Online Published: July 7, 2016

doi:10.5430/bmr.v5n3p1

URL: http://dx.doi.org/10.5430/bmr.v5n3p1

\begin{abstract}
High School teachers in Japan are sending very busy days on their daily works including teaching, support for the club activities and deskwork. Among them, they share a lot of time for managing the club actives of students compared with other countries. In that area, professionals can make instruction much better than teachers for the special sports like Judo and Kendo (Japanese fencing) etc. School Social Worker can coordinate the professionals out of school and can help teachers by decreasing their burden on that area. There are few related papers concerning the support of club activities by utilizing the professionals outside. In this paper, a questionnaire investigation is executed to the four High Schools at Iwate Prefecture in Japan in order to clarify their current condition and their consciousness, and to seek the possibility of utilizing school social worker for their support. Fundamental statistical analysis, Factor Analysis and Multi Correspondence Analysis are performed. Some interesting and instructive results were obtained.
\end{abstract}

Keywords: School Social Worker, High school teacher, Multi Correspondence Analysis, Factor Analysis

\section{Introduction}

Teacher at High school / Junior High School in Japan are sending very busy days in general on their daily works including teaching, support for the club activities and deskwork. Among them, they share a lot of time for managing the club actives of students compared with other countries. In particular, it takes time to manage the club activities for sports. In that area, professionals can make instruction much better than teachers for the special sports like Judo and Kendo (Japanese fencing) etc. School Social Worker can coordinate the professionals out of school and can help teachers by decreasing their burden on that area.

There are many researches made on School Social Workers' function. For example, H. Konyuba (2011) analyzed the teacher's sparing time for club activities and pointed out that there is a difference between the sports club and the culture club. K.Yonekawa (2011) discussed the mental health support by school social worker. M.S.Kelly et al. (2010) made School Social work survey and derived instructive insight.

OECD (Organisation for Economic Co-operation and Development) has released the investigation report of "Teaching And Learning International Survey (TALIS)" on June 25/2014. It is reported that the teacher's total working hours for a week in Japan were the most among 33 countries. Main data are as follows.

/Teacher's total working hours for a week in Japan: 53.9 (Average: 38.3)

/Teacher's working hours of support for the club activities for a week in Japan: 7.7(Average: 2.1)

/Teacher's working hours of deskwork for a week in Japan: 5.5 (Average: 2.9)

/Teacher's teaching hours for a week in Japan: 17.7 (Average: 19.3)

Teacher's working hours of support for the club activities were nearly triple, and those of deskwork double compared with the average, while the teaching hours were less than those of the average. They are too busy for the support of 
the club activities and deskwork, which causes bad influence on their teaching activities.

Although there are some related papers as these, but there are few related papers concerning the support of club activities by utilizing the professionals outside especially in the Japanese case. In particular, Takeyasu et al.(2014) made a research on the possibility of utilizing school social worker to decrease the high school teachers' workload by making Hypothesis Testing. Ishii et al.(2015) analyzed high school teachers' workload in Aomori Prefecture in Japan by making key graph analysis through the questionnaire investigation concerning high school teachers' workload.

In this paper, we further develop this by expanding the research area to another prefecture in Japan. In this paper, a questionnaire investigation is executed to the four High Schools at Iwate Prefecture in Japan in order to clarify their current condition and their consciousness, and to seek the possibility of utilizing school social worker for their support. Fundamental statistical analysis, Factor Analysis and Multi Correspondence Analysis are performed. High school teachers' workload is analyzed through these analyses and the possibility of utilizing school social worker is discussed, which is rarely be seen in the past researches. By this analysis, school social worker's new activation field will be developed.

The rest of the paper is organized as follows. Outline of questionnaire investigation is stated in section 2. Factor Analysis is conducted in section 3 and Multi Corresponding Analysis is executed in section 4 which is followed by the Remarks of section 5.

\section{Outline and the Basic Statistical Results of the Questionnaire Research}

\subsection{Outline of the Questionnaire Research}

We make a questionnaire investigation for the Support of High School Teachers by the School Social Worker. The outline of questionnaire research is as follows.

(1) Scope of investigation : High School Teacher, 4 High Schools in Iwate Prefecture, Japan

(2) Period : January $\sim$ March 2015

(3) Method : Leave until called for

(4) Collection : Number of distribution 120

Number of collection 111(collection rate $92.5 \%$ )

Valid answer 111

\subsection{Basic Statistical Results}

Now, we show the main summary results by single variable.

\subsubsection{Characteristics of Answers (Q4)}

(1) Sex (Q4-1)

Male: $69.37 \%$

Female: $28.83 \%$

(Not filled in: $1.8 \%$ )

(2) Age (Q4-2)

20 29: $10.81 \%$

30 39: $23.42 \%$

40 49: $40.54 \%$

50 59: $19.82 \%$

More than 60: $4.5 \%$

(Not filled in: $0.9 \%$ )

(3) Position (Q4-3)

Deputy Principal: $2.7 \%$

A person in charge of educational affairs: $12.61 \%$

Teacher: $69.37 \%$

Lecturer: $11.71 \%$ 
Assistant: 0.9\%

Miscellaneous: $1.8 \%$

(Not filled in: 0.9\%)

(4) Experience as a teacher (Q4-4)

More than 10 years consists of $64.86 \%$, i.e. nearly $2 / 3$.

(5) How many years are you working for the present school?

5 9 years $(19.82 \%)$ and more than 10 years $(61.26 \%)$ consist of nearly $4 / 5$.

\subsubsection{Club Activities}

(1) Sports Club (Q 1-2-1)

Large part one is Basketball (10.94\%), Baseball (9.38\%) and Tennis (9.38\%).

(2) Culture Club (Q1-2-2)

Main items are as follows.

/ART: $8.33 \%$, /Literature: 6.25\%, /Broadcast Department: 4.17\%, /Theater Photo Department: $4.17 \%$.

(3) How about the sort of job? (Q1-2-2-3)

/Adviser: 77.48\%, /Deputy Adviser: 21.62\%, Miscellaneous: 0.9\%

Adviser holds the majority.

(4) Is the club strong enough to participate in the national sports match? (Q1-2-2-4)

Nearly $1 / 7$ of them are the strong teams to participate in the national sports match.

(5) Is the club activity active? (Q1-2-2-5)

Nearly half of them have selected "Cannot say either".

(6) How long a time do you spare for the club activity (Include moving time) (Q1-2-2-6)

Up to 60 hours in month exceeds $70 \%$ and up to 90 hours reaches $85 \%$ in the total.

2.2.3 Consciousness for the Daily Works (Q2)

(1) Feel it burden to teach (Q2-1)

Nearly $1 / 3$ of the teachers feel it burden to teach.

(2) Feel it burden to prepare for teaching (Q2-2)

Nearly $40 \%$ of the teachers feel it burden to prepare for teaching.

(3) Feel it burden to instruct for learning(Q2-3)

Nearly $31 \%$ of the teachers feel it burden to instruct for learning.

(4) Feel it burden to handle the students' performance(Q2-4)

Nearly $38 \%$ of the teachers feel it burden to handle the students' performance.

(5) Feel it burden to guide students (Q2-5)

Nearly $40 \%$ of the teachers feel it burden to guide students.

(6) Feel it burden to guide club activities(Q2-6)

Nearly $39 \%$ of the teachers feel it burden to guide club activities.

(7) Feel it burden to manage the class $(\mathrm{Q} 2-8)$

Nearly $1 / 3$ of the teachers feel it burden to manage the class.

(8) Feel it burden to deal with meeting, advance arrangement(Q2-9)

Nearly $32 \%$ of the teachers feel it burden to deal with meeting, advance arrangement.

(9) Feel it burden to deal with parents(Q2-10)

Nearly $44 \%$ of the teachers feel it burden to deal with parents. 
2.2.4 Consciousness for Guiding the Club Activities (Q3)

(1) Worthwhile to guide club activities (Q3-1)

Positive attitude to this theme can be seen (52\%).

(2) It is better for the professionals to guide club activities (Q3-2)

Strong positive attitude to this theme can be confirmed (74\%).

(3) I do not know the club field precisely that I take charge of (Q3-3)

Rather many teachers (37\%) feel anxiety for their expertise in their club field.

(4) Quite tired because of the activity on holiday (Q3-5)

Nearly half of them feel tired because of the club activity on holiday.

(5) Have a hard time for the technical guidance (Q3-6)

Nearly half of them have a hard time for the technical guidance.

(6) Have a hard time for the mental guidance (Q3-7)

Nearly $53 \%$ of them have a hard time for the mental guidance.

(7) Want to have a person to consult with in guiding club activities (Q3-8)

Nearly half of them want to have a person to consult with in guiding club activities.

\section{Factor Analysis}

Factor Analysis is executed. As for the extraction method of the factor, "the principal axis factoring" is adopted, and as for the rolling-method, "the varimax rotation which is accompanied by the normalization of kaiser as orthogonal rotation" is adopted. Also, we confirmed the validity of the model in the KMO (Kaisel-Meyer-Olkin) specimen validity measure. When the value is more than 0.8 , it is said meritorious, more than 0.7 middling, more than 0.6 , mediocre. Bartlett sphericity test is executed as well. If the score is less than 0.05 , then it is said that there is a correlation among observation variables.

(1) Q2 "We ask you a consciousness for the daily works"

KMO measure is 0.852 and Bartlett Score is 0.000 . We can confirm a rather appropriate common factor. From the Factor Matrix after rotation (Table 1), we can extract 2 meaningful axes. Summary of factor loading value is $49.155 \%$ therefore extracted 2 factors can explain $49.155 \%$ of the data. We can see that the first axis is the factor about "Teaching and learning for student" as the score for "Teaching", "Preparing Teaching", "Instructing Learning", "Handling Students Performance" and "Student Guidance" are high (Exhibition of characters in the Figure is limited therefore abbreviated expression is used.). Second axis is the factor about "Miscellaneous jobs without Teaching and Learning" as the score for "Club Activities" and "Class Management" "Committee Guidance" and "PTA meeting" are high.

Table 1. Factor Matrix after Rotation

\begin{tabular}{lcc}
\hline & \multicolumn{2}{c}{ Factor } \\
\cline { 2 - 3 } & 1 & 2 \\
\hline Teaching & .718 & .369 \\
PreparingTeaching & .665 & .352 \\
InstructingLaerning & .700 & .317 \\
HnadlingStudentsPerfomance & .414 & .379 \\
StudentGuidance & .396 & .371 \\
ClubActivities & .367 & .534 \\
CommitteeGuidence & .333 & .684 \\
ClassManagement & .178 & .806 \\
ArrangementMeeting & .602 & .154 \\
PTAmeeting & .377 & .539 \\
\hline
\end{tabular}




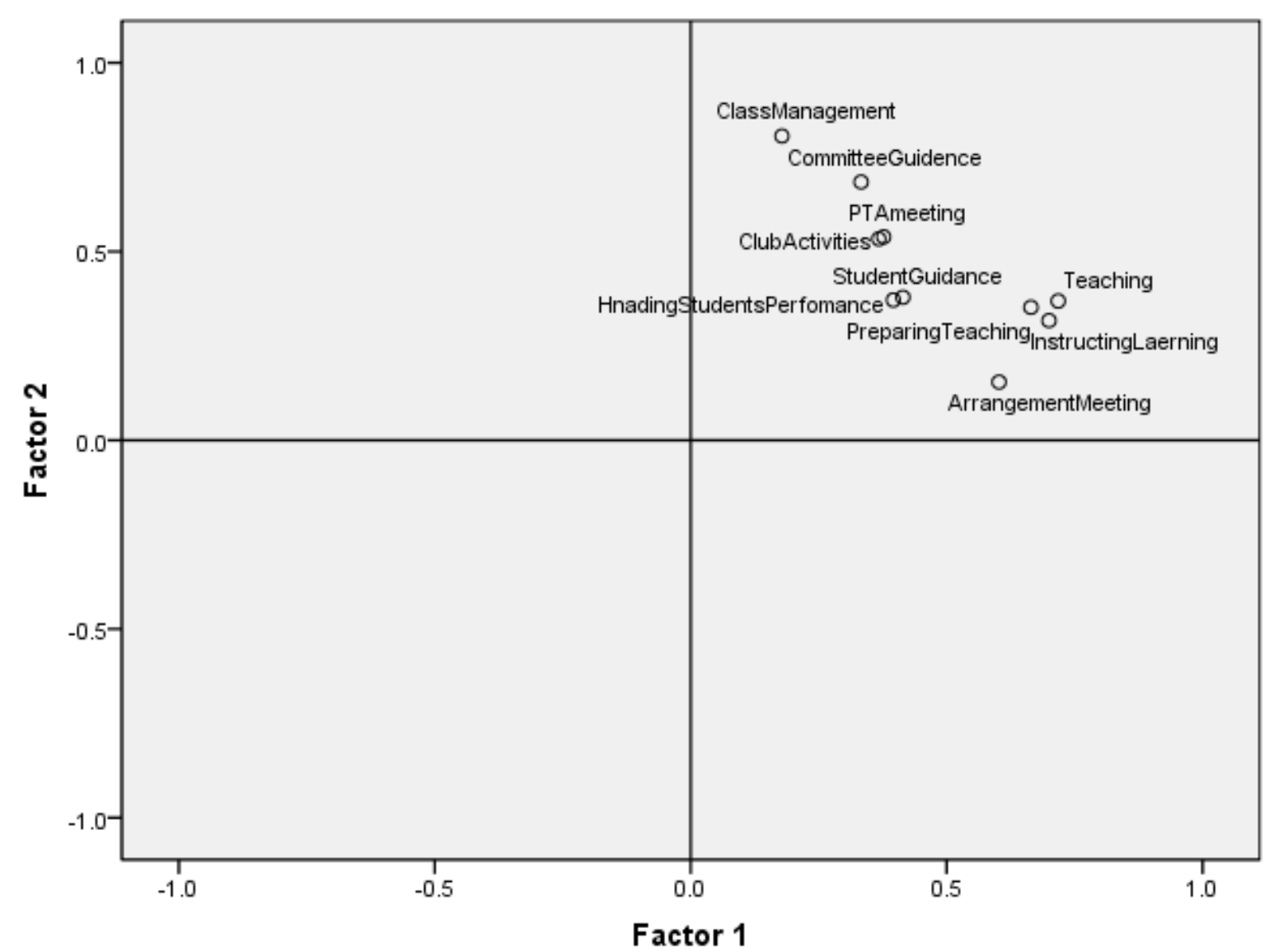

Figure 1. Factor Plotting in Factor Space

(2) Q3 "We ask you a consciousness for guiding the club activities"

KMO measure is 0.796 and Bartlett Score is 0.000 . We can confirm a rather appropriate common factor. From the Factor Matrix after rotation, we can extract 2 meaningful axes. Summary of factor loading value is 39.213\% therefore extracted 2 factors can explain 39.213\% of the data. We can see that the first axis is the factor about "Feel it burden for the club activities" as the score for "Burden for Holiday Activities", Struggling in Technical Guidance", "Cutting Subject Preparation Time", "Want the person to consult with", and "Struggling in Mental Guidance" are high. Second axis is the factor about "Career" as the score for "Feel Worthwhile" and "Inexperience" are high.

Table 2. Factor Matrix after rotation

\begin{tabular}{lrr}
\hline & \multicolumn{2}{c}{ Factor } \\
\cline { 2 - 3 } & 1 & 2 \\
\hline FeelWorthwhile & -.039 & -.508 \\
BetterForTheProfessionals & .245 & .182 \\
Inexperience & .331 & .596 \\
CuttingSubjectPreparationTime & .746 & .148 \\
HolidayActivitiesBurden & .681 & .086 \\
StrugglingTecnicalGuidance & .509 & .314 \\
StrugglingMentalGuidance & .520 & .121 \\
WantPeopleWhoCanConsult & .724 & .320 \\
\hline
\end{tabular}




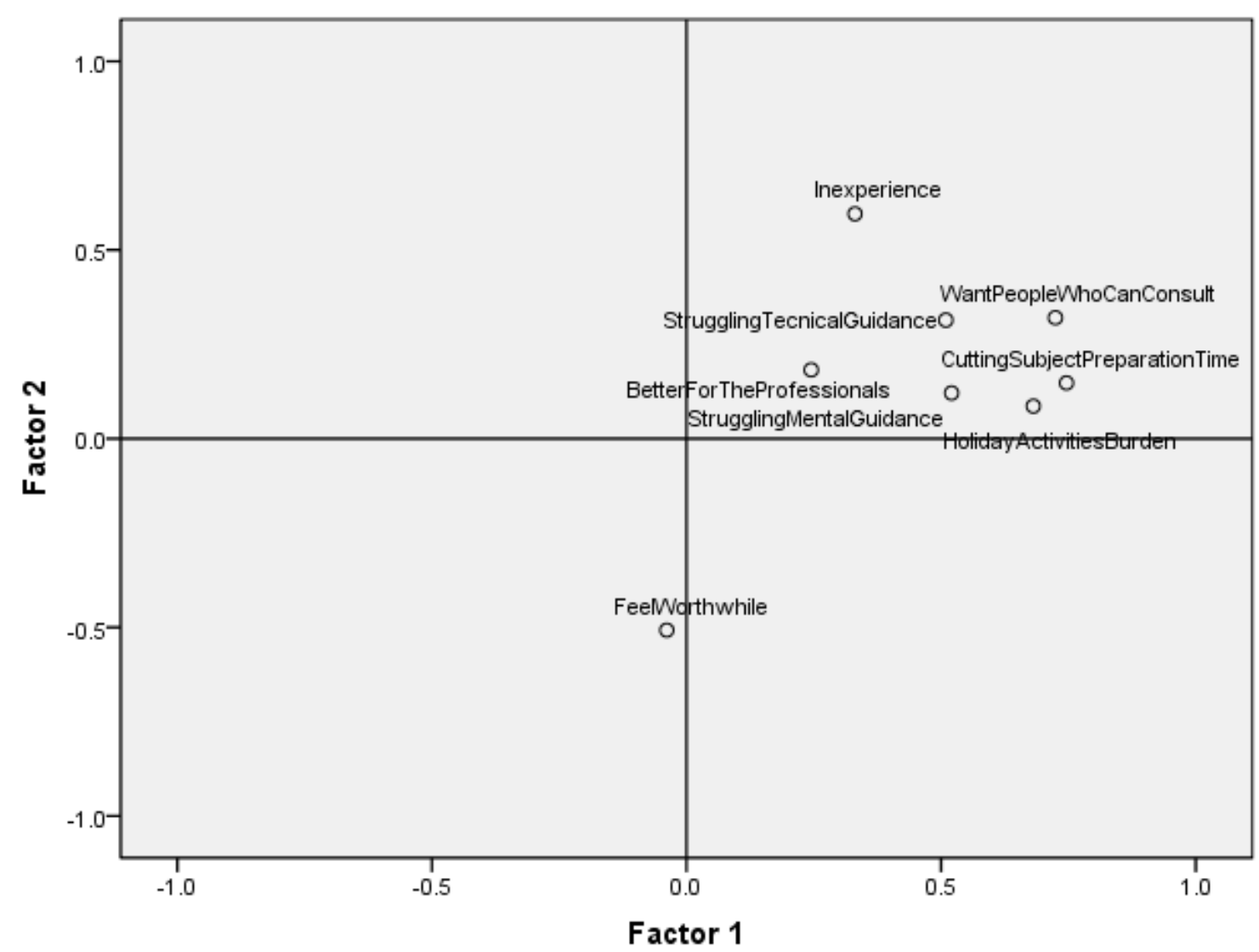

Figure 2. Factor Plotting in Factor Space

In the above Table, there was no hatched part in "Better for the professionals to Guide" therefore we skip this part and check it again without this.

KMO measure is 0.808 (nearly the same with before) and Bartlett Score is 0.000 . We can confirm a rather appropriate common factor. From the Factor Matrix after rotation, we can extract 2 meaningful axes. Summary of factor loading value is $43.657 \%$ therefore extracted and it can be said that the relation among items became much clearer. The meaning of the first axis is basically the same with before. While the second axis is the factor about "Negative Career" as the score for "Inexperience" is high.

Table 3. Factor Matrix after rotation

\begin{tabular}{lcr}
\hline & \multicolumn{2}{c}{ Factor } \\
\cline { 2 - 3 } & 1 & 2 \\
\hline FeelWorthwhile & -.047 & -.479 \\
Inexperience & .322 & .638 \\
CuttingSubjectPreparationTime & .758 & .162 \\
& & \\
HolidayActivitiesBurden & .670 & .077 \\
StrugglingTecnicalGuidance & .505 & .298 \\
& & \\
StrugglingMentalGuidance & .528 & .125 \\
WantPeopleWhoCanConsult & .720 & .316 \\
\hline
\end{tabular}




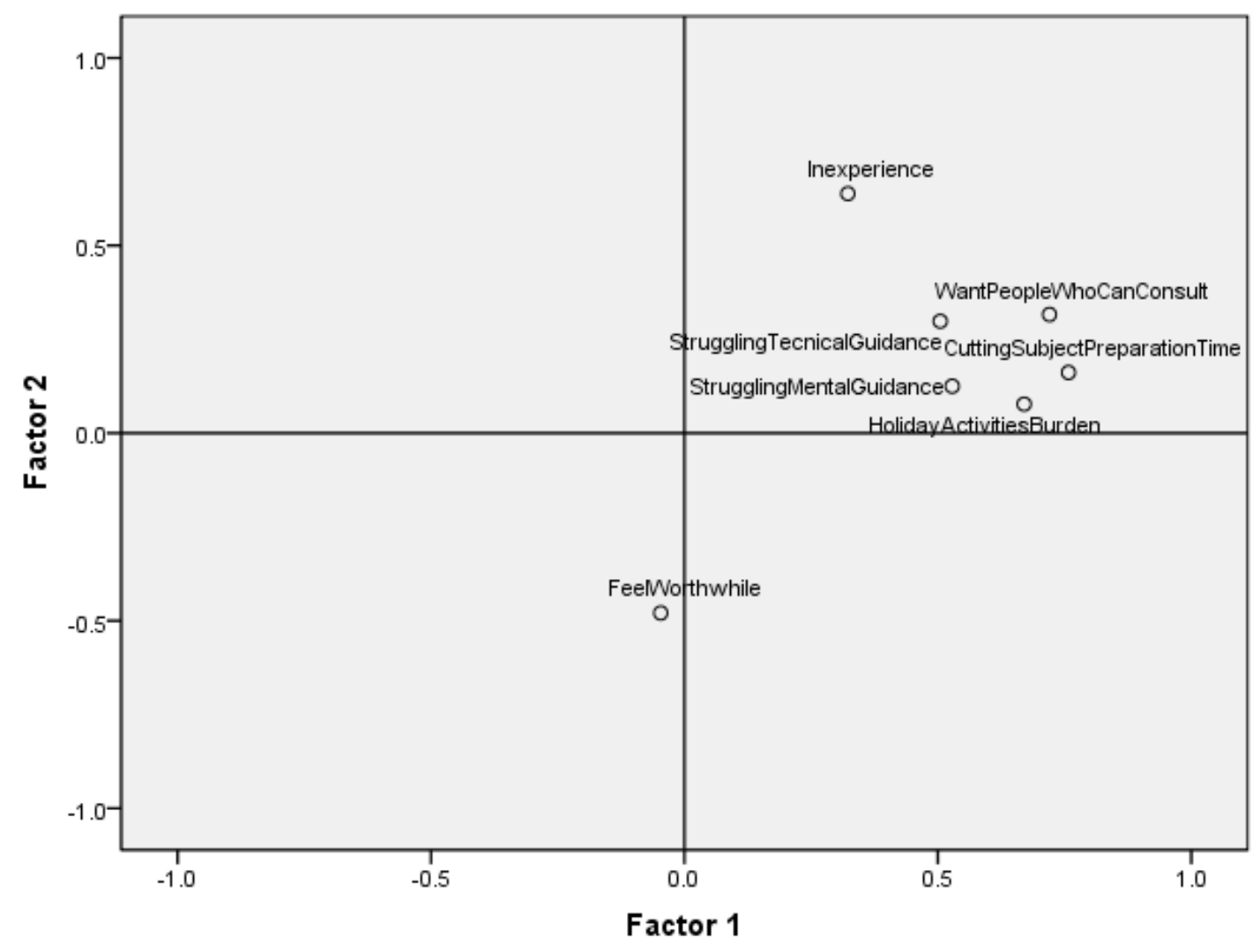

Figure 3. Factor Plotting in Factor Space

\section{Multi Correspondence Analysis}

We execute the multi correspondence analysis in Q2 "We ask you a consciousness for the daily works" and Q3 "We ask you a consciousness for guiding the club activities" in order to find the correlation of each items.

(1) Q2 "We ask you a consciousness for the daily works"

We can observe the following result from Figure 4. From the data, an eigenvalue of Dimension 1 axis is 0.486 and those of Dimension 2 is $0.321 .80 .7 \%$ can be explained by these two major axes. We can extract 4 clusters. We can assume that the first cluster (Right Upper ) shows "Teaching and Learning for students" (Teaching, Preparing Teaching, Instructing Learning, Committee Guidance). The second one(Left Upper) would mean "Management Activity except for teaching" (Meeting, PTA meeting). The third one (Left Center) is about "Student guidance on student performance" (Handling Student Performance, Student Guidance). The fourth one (Left Lower) would be "Educational Activity except for teaching"(Class Management, Club Activity).

Among them, the cluster "Club Activities" is far from other clusters and it shows the uniqueness of its characteristics. 


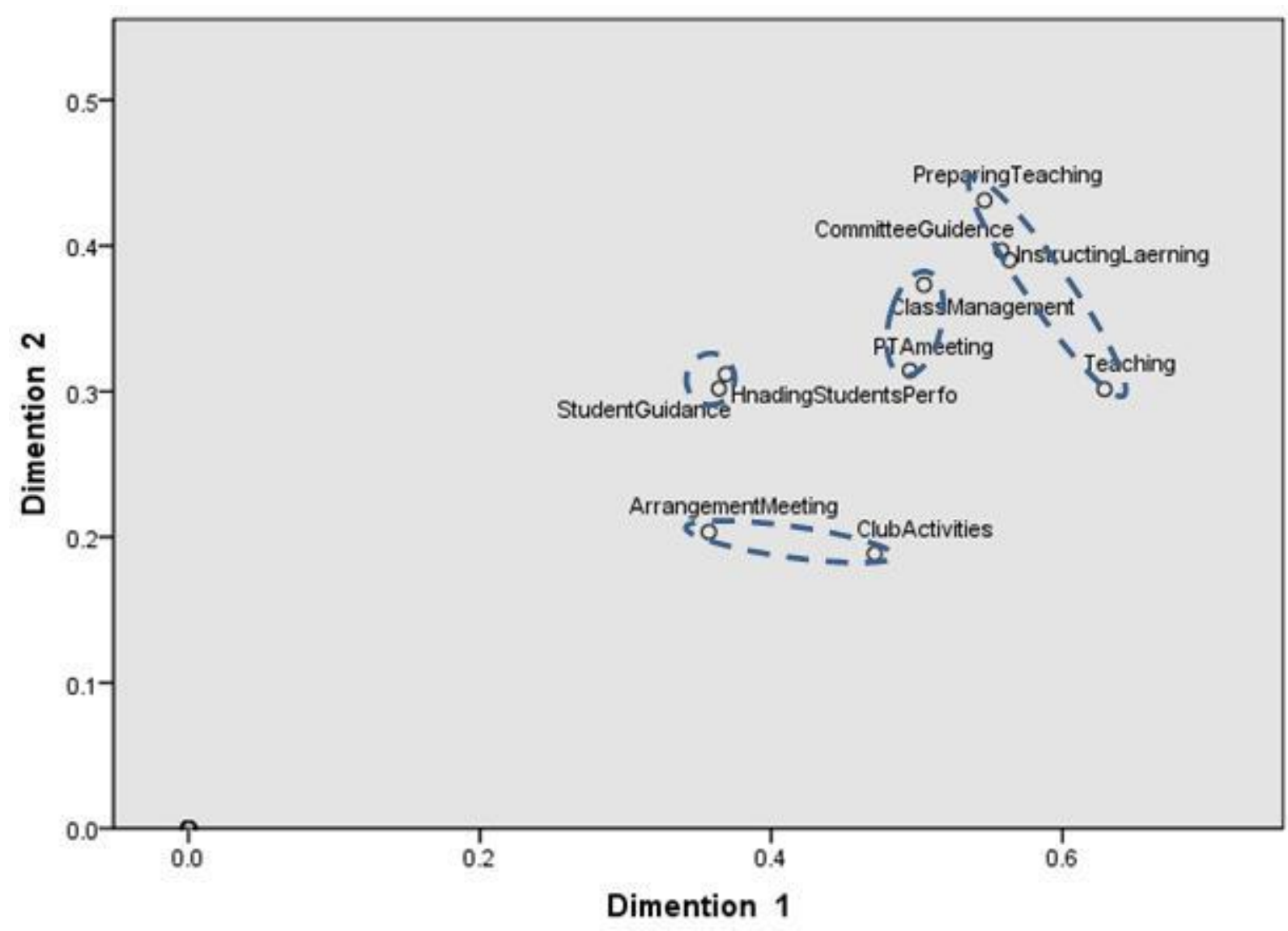

Figure 4. Q2 Consciousness for the daily works

(2) Q3 "We ask you a consciousness for guiding the club activities"

We can observe the following result from Figure 5. From the data, an eigenvalue of Dimension 1 axis is 0.413 and those of Dimension 2 is 0.259 . We can extract 4 clusters. We can assume that the first cluster (Right Upper ) shows "Hardness in guiding" (Struggling in Technical Guidance, Struggling in Mental Guidance). The second one(Right Middle) would mean "Mental burden" (8.Want the person to consult with). The third cluster (Left Middle) would mean "Lack of Specialization" (Better for the professionals to Guide). The other cluster maybe "Career of himself/herself" ( Inexperience, Feel worthwhile, Cutting Subject Preparation Time, Burden for Holiday Activities). 


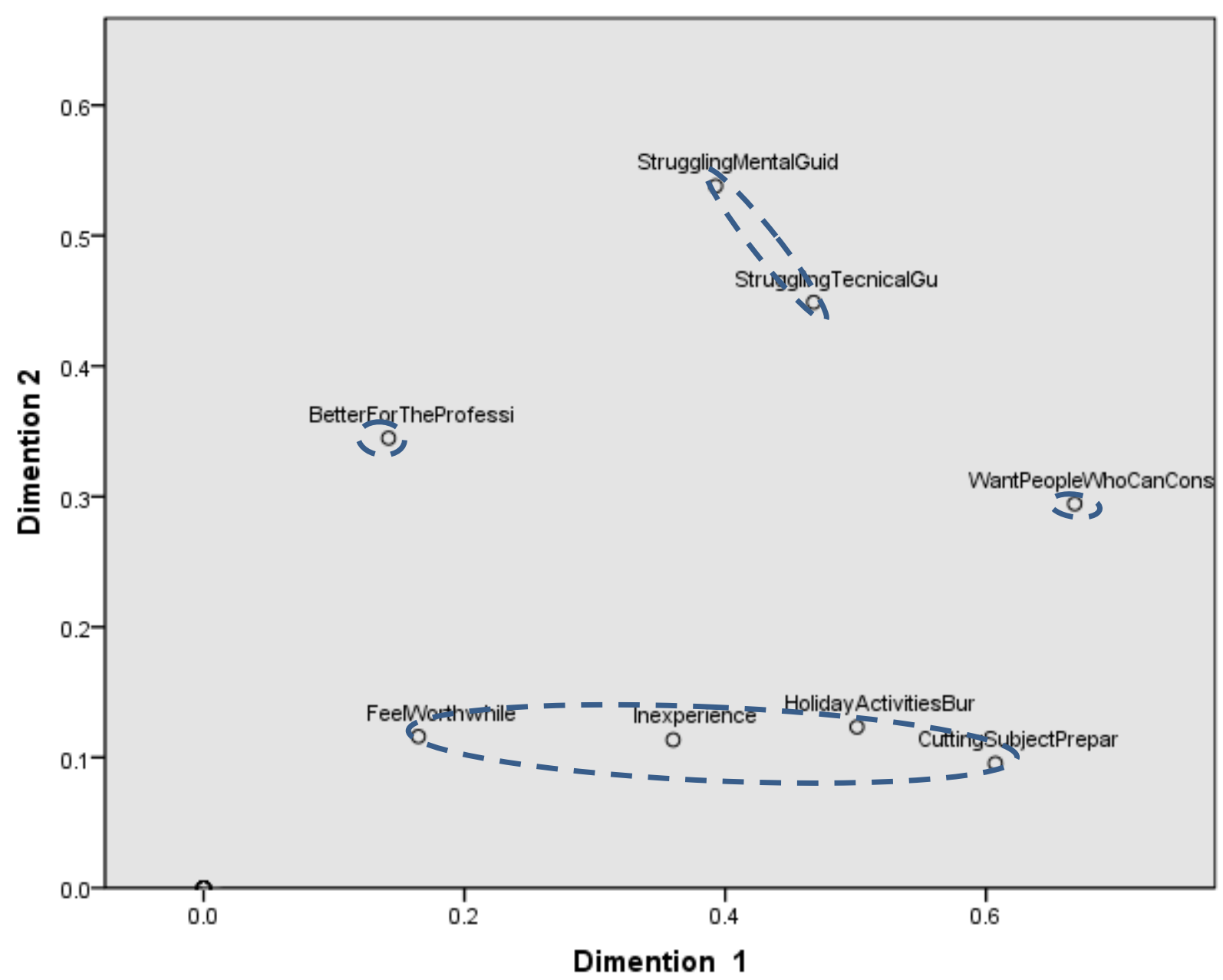

Figure 5. Q3 Consciousness for guiding the club activities

\section{Remarks}

In this paper, a questionnaire investigation is executed in order to clarify their current condition and their consciousness, and to seek the possibility of utilizing school social worker for their support.

In the Factor Analysis, 2 meaningful axes could be extracted in the consciousness for the daily works and also 2 meaningful axes could be extracted in the consciousness for guiding the club activities. In the Multi Correspondence Analysis, 4 clusters could be confirmed. Among them, in the consciousness for the daily works, the cluster "Club Activities" and "Meeting" consisted as an independent item and was located far from other clusters and it shows the uniqueness of its characteristics. In the consciousness for guiding the club activities, "Want the person to consult with" and "Better for the professionals to Guide" located as an independent item and was located far from other clusters and it shows the uniqueness of its characteristics.

Considering these facts, these suggest that unique/original approach should be executed to the "Club Activities". Based upon the results of hypothesis testing, teachers' burden may be decreased by utilizing outer specialist in guiding club activities. School Social Worker can coordinate the professionals out of school and can help teachers by decreasing their burden on that area. This suggests the possibility of developing the new activity field for the School Social Worker. By this analysis, school social worker's new activation field will be developed.

\section{Conclusion}

High School teachers in Japan are sending very busy days on their daily works including teaching, support for the club activities and deskwork. Among them, they share a lot of time for managing the club actives of students compared with other countries. In that area, professionals can make instruction much better than teachers for the special sports like Judo and Kendo (Japanese fencing) etc. School Social Worker can coordinate the professionals out 
of school and can help teachers by decreasing their burden on that area. There are few related papers concerning the support of club activities by utilizing the professionals outside. In this paper, a questionnaire investigation was executed to the four High Schools at Iwate Prefecture in Japan in order to clarify their current condition and their consciousness, and to seek the possibility of utilizing school social worker for their support. Fundamental statistical analysis, Factor Analysis and Multi Correspondence Analysis were performed.

In the Multi Correspondence Analysis, the following two unique results could be obtained. In the consciousness for the daily works, the cluster "Club Activities" and "Meeting" consisted as an independent item and was located far from other clusters and it shows the uniqueness of its characteristics. In the consciousness for guiding the club activities, "Want the person to consult with" and "Better for the professionals to Guide" located as an independent item and was located far from other clusters and it shows the uniqueness of its characteristics.

Based upon the results, these suggest that unique/original approach should be executed to the "Club Activities". Based upon the results of hypothesis testing, teachers' burden may be decreased by utilizing outer specialist in guiding club activities. School Social Worker can coordinate the professionals out of school and can help teachers by decreasing their burden on that area. This suggests the possibility of developing the new activity field for the School Social Worker. By this analysis, school social worker's new activation field will be developed. Yet, there still remains the future works that much more cases should be executed on this to confirm this proposition. Further study on this should be executed such as text mining analysis. Various cases should be investigated here after.

\section{Acknowledgements}

The authors are grateful to all those who supported us for answering questionnaire investigation.

\section{References}

Bennesse Education Research Institute. (2010). The 5th Basic Investigation Report about Instruction for Learning (Elementary School, Junior High (http://benesse.jp/berd/center/open/report/shidou_kihon5/sc_hon/index.html )

Bennesse Education Research Institute. (2010). The 5th Basic Investigation Report about Instruction for Learning (High School). (http://benesse.jp/berd/center/open/report/shidou_kihon5/kou_hon/index.html)

Daisuke Takeyasu, Tatsuya Oyanagi, Kazuhiro Takeyasu. (2014). Questionnaire Investigation for the Support of High School Teachers by the School Social Worker. Journal of Computations \& Modelling, 4(4), 1-52.

Hideyuki Konyuba. (2011). Analysis on teachers' workload and development of school organization: focusing on the school club activities. National Institute for Educational Policy Research, 140, 181-193.

Kazuo Yonekawa. (2011). The role of school social worker for mental health of a junior high school teacher, Bulletin of Faculty of Literature, Kurume University, 10*11, 7-15.

Michael Stokely Kelly. (2010). Stephanie Cosner Berzin, Andy Frey, Michelle Alvarez, Gary Shaffer and Kimberly O'Brien, "The State of School Social Work: Findings from the National School Social Work Survey", School Mental Health, September 2010, Volume 2, Issue 3, pp132-141. http://dx.doi.org/10.1007/s12310-010-9034-5

Yasuo Ishii, Tatsuya Oyanagi, Daisuke Takeyasu, Kazuhiro Takeyasu. (2015). Text Mining Analysis for the Questionnaire Investigation Concerning High School Teachers' Work Load. Journal of Computations \& Modelling, 5(4), 41-74. 